\title{
MARCADORES SONOGRAFICOS DE CARDIOPATIAS CONGENITAS. PERSISTENCIA DE LA VENA CAVA SUPERIOR IZQUIERDA: NUESTRA EXPERIENCIA*
}

\author{
Drs. Fernando Viñals L. y Arrigos Giuliano B. \\ Centro AGB Ultrasonografía. Clínica Sanatorio Alemán, Concepción
}

\section{RESUMEN}

Consideramos marcadores de cardiopatías congénitas al grupo de lesiones que, pudiendo ser variantes normales, se asocian a defectos cardíacos. En esta segunda publicación destacamos la importancia del diagnóstico de la persistencia de la vena cava superior izquierda y su drenaje hacia el seno coronario, como un marcador de cardiopatías congénitas. En nuestra serie de cardiopatías congénitas (Cc), hemos diagnosticado 5 persistencias de la vena cava superior izquierda, 4 de ellas drenando hacia el seno coronario; 2 fueron anomalías aisladas, correspondiendo a variantes anatómicas. En otros 2 casos, los fetos eran portadores de cardiopatías congénitas mayores, uno de ellos actualmente vivo después de corrección quirúrgica y el restante fallecido. El quinto caso era un feto portador de anomalías múltiples, sospechándose una cromosomopatía, la que fue confirmada postparto como una trisomía 13. Reportamos los hallazgos in útero, el resultado postnatal de estos casos, la técnica más apropiada para su pesquisa y su asociación a Cc.

\section{PALABRAS CLAVES: Cardiopatías congénitas, marcadores sonográficos}

\section{SUMMARY}

We consider markers of congenital heart disease a group of anomalies which could be a normal variant in general population but might be associated to structural defects of the fetal heart. One of those markers is the persistence of the left superior vena cava and his drainage to the coronary sinus. Among 5 persistence of the left superior vena cava diagnosed in our series, we found 2 associated with major congenital heart disease and another one with abnormal karyotype (trisomy 13). 2 other fetus were diagnosed as an isolated lesion. We discuss in utero abnormalities and fllow-up. We considered that the diagnosis of persistence of the left superior vena cava is a tool for the in utero detection of congenital heart disease.

\section{KEY WORDS: Left superior vena cava, coronary sinus, congenital heart disease, prenatal diagnosis}

\section{INTRODUCCION}

Definiremos como marcadores sonográficos de cardiopatías congénitas (Cc) aquel grupo de lesio- nes que, pudiendo ser variantes normales en la población general, se asocian a defectos estructurales cardíacos. El hallazgo ecográfico de estos permite seleccionar aquellos fetos que requerirán

*Trabajo recibido en julio de 2002 y aceptado para publicación por el Comité Editor en septiembre de 2002. 
un estudio ecocardiográfico detallado, además de un apropiado consejo. En este segundo trabajo nos referiremos a la presencia de 2 venas cavas superiores que drenan al corazón fetal en vez de una.

Desde un punto de vista embriológico, 3 pares de venas drenan hacia el corazón del embrión de 4 semanas:

1. Las venas vitelinas que retornan la sangre pobremente oxigenada del saco vitelino.

2. Las venas umbilicales que transportan sangre oxigenada desde las vellosidades coriales; de estas sólo la izquierda persiste.

3. Las venas comunes cardinales que drenan sangre poco oxigenada del cuerpo del embrión.

Estas últimas constituyen el principal sistema de drenaje venoso del embrión. Las venas cardinales anterior y posterior drenan respectivamente la porción craneal y caudal del embrión. Estas se unen en las venas cardinales comunes que drenan al seno venoso (también denominado seno coronario). La vena cava superior se desarrolla desde la vena cardinal derecha anterior y la vena común cardinal derecha. La porción caudal de la vena cardinal izquierda anterior degenera alrededor de las 8 semanas del desarrollo embrionario (1) (Figura 1).

Producto de la serie de transformaciones del sistema venoso a este nivel, pueden observarse variantes anatómicas en la forma adulta. Una de las más frecuentes la persistencia de la vena cava superior izquierda (VCSi). Esta vena anormal deriva de la vena cardinal anterior izquierda y las venas cardinales comunes. Se conecta clásicamente a la aurícula derecha a través del seno coronario. En estos casos, el seno se encuentra dilatado hasta 3 veces su tamaño normal y drena en la aurícula derecha (2). En aquellos casos en que se conecta a la aurícula izquierda, habitualmente el seno no es visible ecográficamente.

La persistencia de la VCSi ha sido observada en un $0,3-0,5 \%$ de la población general, sin determinar consecuencia funcional alguna $(3,4)$. Sin embargo, $2-10 \%$ de las cardiopatías congénitas (Cc) se asocian a una persistencia de la VCSi $(4,5)$. También se ha asociado a anomalías cromosómicas como la trisomía 13 y 18. Por este motivo, su reconocimiento es importante y constituye una herramienta más para la sospecha diagnóstica de cardiopatías congénitas durante la vida antenatal.

Nuestro interés es describir cómo puede diagnosticarse la persistencia de la VCSi y su drenaje al seno coronario, nuestros casos y su interpretación clínica.

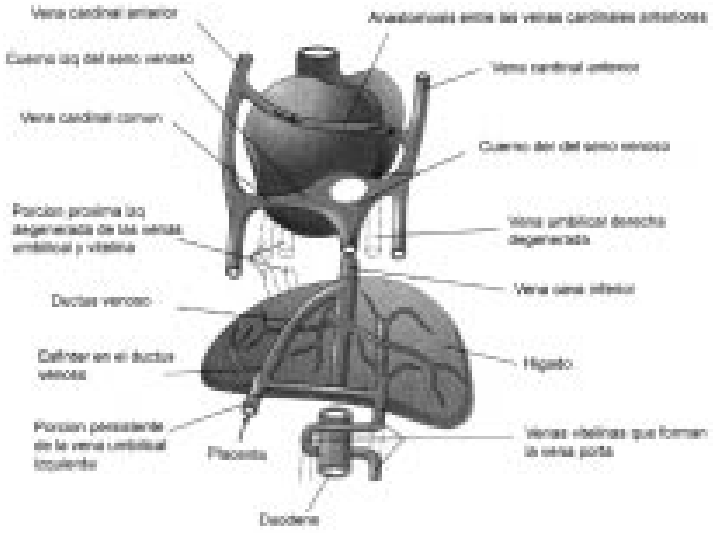

Figura 1. Esquema del desarrollo embriológico a las 7 semanas de vida intrauterina del seno venoso y la circulación venosa del hígado.

\section{MATERIAL Y METODO}

Las pacientes evaluadas en nuestro Centro, predominantemente de bajo riesgo de dar a luz recién nacidos (RN) con cardiopatía congénitas (Cc), son examinadas ecográficamente adicionando un estudio extendido del corazón fetal que incluye una secuencia de secciones transversas desde el abdomen fetal hasta el mediastino superior, en blanco y negro y adicionando Doppler color, según técnica descrita previamente $(6,7)$. En caso de sospecharse una alteración estructural del corazón, se efectuaron inmediatamente las secciones ecocardiográficas necesarias para completar su estudio. Se obtuvo un respaldo digital de todos los casos de Cc diagnosticadas para su estudio y análisis posterior.

Del total de casos de Cc diagnosticados in utero en nuestra serie, seleccionamos aquellos en los cuales se consignó la persistencia de la VCSi, ya sea que esta anomalía fuera aislada o asociada a otras alteraciones estructurales del corazón fetal o extracardíacas.

Técnicamente, los planos de sección más importantes para la búsqueda de este marcador se encuentra en el mediastino superior fetal. A esta región se accede ecográficamente deslizando el transductor desde la visión de 4 cámaras hacia cefálica fetal, sin angularlo. Esto obliga a que el operador tenga una idea apropiada de la "variedad de posición" fetal al momento de la exploración. Las secciones a destacar son la visión de 3 vasos (8) y la visión de arco aórtico transverso (9). Es importante recordar los reparos anatómicos de ambos. En la visión de 3 vasos, algo más hacia cefálica 
desde las 4 cámaras, se visualizan de izquierda a derecha fetal 3 estructuras vasculares: el tronco de la arteria pulmonar hasta su bifurcación, el arco aórtico ascendente y la vena cava superior (derecha). Esto 2 últimos vasos se visualizan en sección oblícua. Habitualmente, entre las estructuras arteriales y la venosa se observa la vía aérea. Si el transductor se desliza ligeramente hacia cefálica, la arteria pulmonar se continua con el ductus arterioso, el arco aórtico se visualiza en su porción transversa y la vena cava superior es de características similares a la proyección anterior. En este plano, denominado visión de arco aórtico transverso, los dos vasos arteriales se disponen en forma oblicua dirigiéndose hacia la columna vertebral fetal. Los reparos anatómicos de ambos planos se presentan en la Figura 2.

En condiciones fisiológicas, en ambos planos de sección se observan 3 vasos y la vía aérea. Una de las anomalías posibles de encontrar a este nivel es el mayor número de vasos. La causa más frecuente es la persistencia de la vena cava superior izquierda. En estos casos, un cuarto vaso de calibre similar al de la vena cava superior derecha se observa a izquierda y algo más posterior de la arteria pulmonar. Cuando se trata de una variante anatómica con fisiológica cardiovascular normal, en ausencia de defectos cardíacos asociados, 4 vasos son fácilmente pesquisables en la sección de 3 vasos y en arco aórtico transverso. En caso de ser la persistencia una alteración más de la anatomía cardíaca, diversas anomalías de posición pueden observarse en el mediastino superior fetal (8).

En caso de existir una persistencia de la VCSi, habitualmente su drenaje es al seno coronario. Al recibir este el retorno venoso extra, se dilata, pudiendo visualizarse fácilmente hacia la base de la aurícula izquierda fetal. Su detección es posible mediante una prolija evaluación de las 4 cámaras (6). Considerando que sistemáticamente es la visión de 4 cámaras el primero de los pasos para la
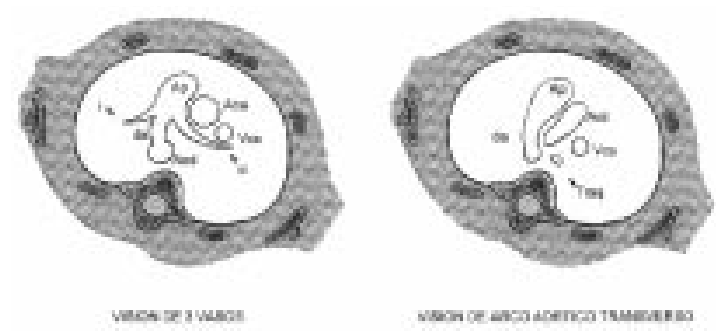

Figura 2. Esquemas sobre reparos anatómicos del mediastino superior fetal.

evaluación del corazón fetal, la visualización del seno coronario dilatado es el marcador de la persistencia de la VCSi.

\section{RESULTADOS}

Del total de fetos con Cc diagnosticados en nuestro Centro desde 1999 hasta la fecha, 5 fueron informados como portadores de una VCSi. En 4 de ellos se confirmó además el drenaje hacia el seno coronario. Los detalles de los casos se presentan en las Tablas I y II.

De los 5 casos de persistencia de la VCSi, 2 fueron recién nacidos sanos, asintomáticos hasta el momento de la publicación. Otros 2 fetos eran portadores de Cc mayores, uno de ellos vivo e intervenido quirúrgicamente de una comunicación perimembranosa amplia con aorta cabalgante y sin estenosis pulmonar. El otro debutó clínicamente con una bradiarritmia. Fue derivada a nuestro Centro por este motivo, pesquisándose una transposición corregida de las grandes arterias (L-TGA). El recién nacido evolucionó desfavorablemente postparto, falleciendo producto de una bronconeumonía. Uno de los casos correspondió a un feto portador de una trisomía 13. Fue derivado por oligoamnios a las 33 semanas de gestación, por lo cual la evaluación morfológica fetal fue parcial. A nivel cardíaco no se observaron defec-

Tabla I

DISTRIBUCION DE CASOS DE PERSISTENCIA DE LA VENA CAVA SUPERIOR IZQUIERDA Y DRENAJE AL SENO CORONARIO

\begin{tabular}{lcllccc}
\hline Caso & Edad & Motivo US & $1^{\text {er US/Control }}$ & EG diagnóstico & VCSI & Seno coronario \\
\hline 1 & 18 & Control & Seguimiento & $25 \mathrm{~s}$ & Sí & Sí \\
2 & 32 & Control & Seguimiento & $34 \mathrm{~s}$ & Sí & Sí \\
3 & 38 & Control & $1^{\text {er US }}$ & $23 \mathrm{~s}$ & Sí & Sí \\
4 & 39 & Oligoamnios & $1^{\text {er US }}$ & $33 \mathrm{~s}$ & Sí & Sí \\
5 & 23 & Arritmia & $1^{\text {er US }}$ & $26 \mathrm{~s}$ & Sí & - \\
\hline
\end{tabular}

US: Ultrasonido; EG: Edad gestacional (semanas). 
Tabla II

ANOMALIAS CARDIACAS Y EXTRACARDIACAS EN CASOS DE PERSISTENCIA DE LA VCSi. RESULTADO POSTNATAL DE LOS CASOS

\begin{tabular}{lccl}
\hline Caso & VCSi/Sc & Anomalía cardíaca/extracardiaca & Resultado \\
\hline 1 & Sí/sí & No & RN vivo-asintomático \\
2 & Sí/sí & CIV mal alineada - sin estenosis pulmonar & RN vivo-operado-bien \\
3 & Sí/sí & No & RN vivo-asintomático \\
4 & Sí/sí & RCIU-OHA-pieloectasia-hipospadia-fisura labial & T13-muerte neonatal \\
5 & Sí/- & L-TGA-Civ-bloqueo AV incompleto & RN vivo-muerte por bronconeumonía \\
\hline
\end{tabular}

RN: Recién nacido; CIV: comunicación interventricular; RCIU: retraso del crecimiento intrauterino; OHA: oligoamnios; L-TGA: transposición corregida de los grandes vasos; AV: aurículoventricular. T13: trisomía 13.

tos estructurales mayores posibles de diagnosticar en esas condiciones, destacando una desproporción marcada de las cavidades ventriculares (dominancia derecha), una persistencia de la VCSi y su drenaje al seno coronario.

\section{DISCUSION}

La persistencia de la vena cava superior izquierda (VCSi) es la anomalía más común de la vena cava superior. Se ha reportado en $0,3 \%$ de 4000 autopsias no seleccionadas (10). La VCSi persistente se conecta a la aurícula derecha en el $92 \%$ de los casos, a través del seno coronario. En el restante $8 \%$, su conexión es hacia la aurícula izquierda (11).

El seno coronario normal drena sangre venosa de las venas cardíacas a la aurícula derecha. En algunas circunstancias, el seno coronario está dilata-
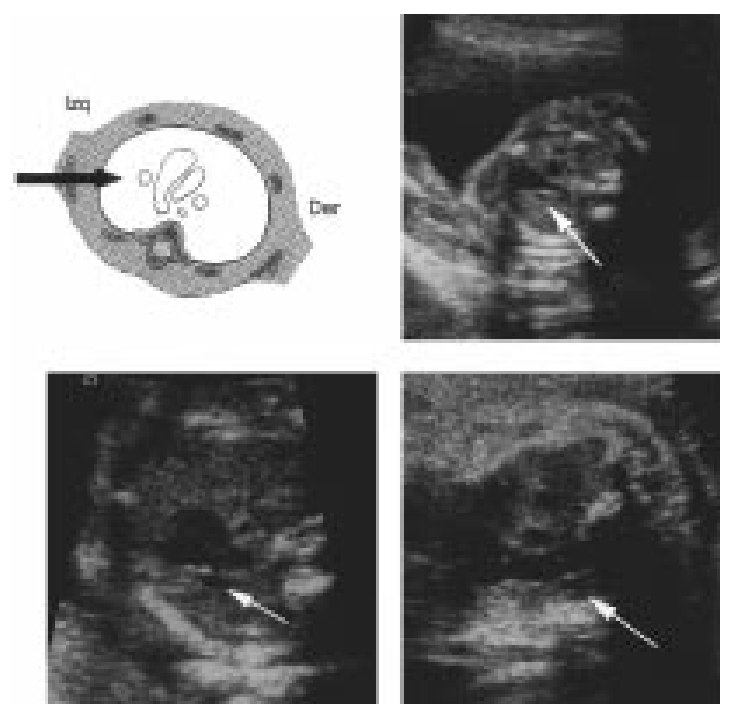

Figura 3. Esquema y casos de persistencia de la vena cava superior izquierda fetal (flecha). do, ya sea por sobrecarga de volumen o raramente de presión. El seno se localiza adyacente y ligeramente posterior al anillo de la válvula mitral. En condiciones fisiológicas, su visualización es fácil en la visión apical de 4 cámaras, la más sencilla de reproducir de las secciones cardíacas y la más utilizada para la pesquisa de Cc en el segundo trimestre. En un trabajo reciente de Rein y cols. (2), todos los casos en que estaba dilatado se relacionaron con persistencia de la vena cava superior izquierda que drenaba a este seno. La mayoría de las dilataciones fue evidente desde las 22 semanas de gestación. Se visualizó como un área ovalada y econegativa ubicada entre la aurícula izquierda. Por este motivo, el hallazgo más fácil de reconocer en los casos de persistencia de la VCSI con conexión al seno coronario es la presencia del seno dilatado (Figura 4). Este puede ser identificado en la visión de 4 cámaras, enfocando hacia posterior e inferior del corazón, justo por encima del velo posterior de la válvula mitral. En ausencia de VCSI persistente, el seno coronario puede visualizarse, pero es significativamente de menor tamaño (2).

La simple falla en la obliteración de la vena cardinal anterior izquierda resulta en la persistencia de la vena cava superior izquierda. En estos casos,
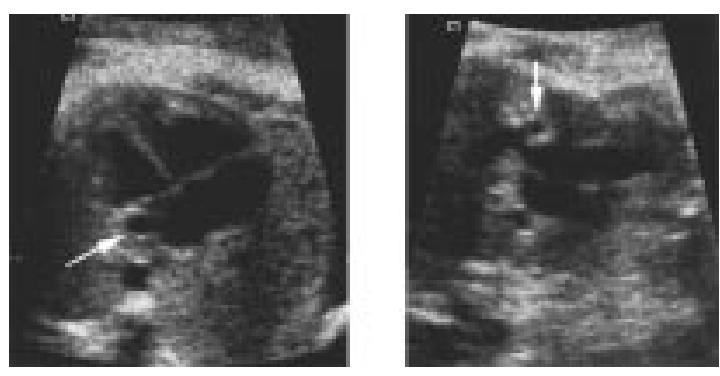

Figura 4. Seno coronario dilatado y persistencia de la VCSi en un caso de comunicación interventricular mal alineada sin estenosis pulmonar. 
el seno coronario sirve de conducto entre la VCSi y la aurícula derecha. En ausencia de defectos cardíacos asociados, la fisiología es normal. Sin embargo, su importancia está en la frecuente asociación a otras malformaciones cardíacas congénitas como cor tritriatum, atresia mitral, cardiopatías congénitas cianóticas como la tetralogía de Fallot y defectos auriculoseptales de tipo seno venoso (12, 13). Su presencia es habitual en anomalías por malposición visceral (14). En relación a estas últimas, la presencia de 2 venas cavas superiores bilaterales es un hallazgo de relativa frecuencia en casos de isomerismo auricular. Se asocia aproximadamente en un $61 \%$ de los casos a isomerismo derecho y en un $56 \%$ a isomerismo izquierdo (150. Sin embargo, la conexión de la VCSi es diferente en cada uno de ellos. En los casos de isomerismo derecho, la conexión es directa hacia la aurícula morfológicamente derecha. La presencia de una persistencia de la VCSi puede además producir complicaciones técnicas durante la ecocardiografía, la cateterización cardíaca o la cirugía cardíaca $(1,3)$.

En ausencia de enfermedad cardíaca asociada, este cuadro no representa riesgo para el feto o recién nacido. Posterior a la evaluación ecocardiográfica y habiéndose descartado defectos cardíacos asociados, el parto puede programarse en cualquier centro. Es importante efectuar una ecocardiografía postnatal con el fin de descartar un defecto septal tipo seno coronario, aunque esta lesión no produce habitualmente síntomas en la infancia. La presencia de una persistencia de la VCSI puede sí complicar la corrección quirúrgica de cardiopatías complejas, por lo que esto debe considerarse al momento del consejo antenatal (14).

El hallazgo ecográfico de una vena cava superior izquierda persistente y su continuación al seno coronario es un marcador de cardiopatías congénitas. Esta anomalía determina alteraciones fáciles de pesquisar cuando se aplica una técnica secuencial de exploración del corazón fetal. El hallazgo de este defecto obliga a efectuar un estudio ecocardiográfico fetal y entregar un apropiado consejo a la pareja. Debe considerarse como otra herramienta más para incrementar la detección antenatal de cardiopatías congénitas.

\section{BIBLIOGRAFIA}

1. Moore KL, Persaud TVN: The developing human. Clinically oriented embriology. $6^{\text {th }}$ ed. Phihladelphia: WB Saunders y Co 1998; 349-60.
2. Rein AJ, Nir A, Ndjari M: The coronary sinus in the fetus. Ultrasound Obstet Gynecol 2000; 15: 468-72.

3. Biffi M, Boriani G, Frabeti L, Bronzetti G, Branzi A: Left superior vena cava persistence in patients undergoing pacemaker or cardioverter-defibrilator implantation: a 10 year experience. Chest 2001; 120 : 139-44.

4. Perloff JK: Congenital anomalies of vena caval connection. In: The Clinical Recognition of Congenital Heart Disease $4^{\text {th }}$ ed. WB Saunders \& Co 1994ñ 70314.

5. Nsah EN, Moore GW, Hutchins GM: Pathogenesis of persistent left superior vena cava with a coronary sinus connection. Pediatr Pathol 1991; 11: 261-69.

6. Viñals F, Giuliano A: Exploración del corazón fetal: ¿Cómo simplificar las cosas? ReV CHIL OBStet GINECOL 1999; 4: 275.

7. Viñals F, Tapia J, Giuliano A: Diagnóstico antenatal de defectos ventrículoseptales. A propósito de nuestra técnica, experiencia y consideraciones perinatales. Rev Chil Obstet Ginecol 2000; 3: 177-82.

8. Yoo SJ, Lee YH, Kim ES, Ryu HM, Kim MY, Choi HK, Cho KS, Kim A: Three-vessel view of the fetal upper mediastinum: an easy means of detecting abnormalities of the ventricular outflow tracts and great arteries during obstetric screening. Ultrasound Obstet Gynecol 1997; 9: 173-83.

9. Allan LD: The normal fetal heart. In: Allan L, Hornberger L, Sharland G. Textbook of Fetal Cardiology. London: Greenwich Medical Media Limited 2000; 55102.

10. Krabill KA, Lucas RV: Abnormal pulmonary venous connections. In: Emmanouilides GC, Riemenschneider TA, Allen HD, Gutgesell HP (eds). Moss and Adams. Heart Disease in Infants, Children and Adolescents. 5th ed. Baltimore: Williams and Wilkins 1995; 874-5.

11. Meadows WR, Sharp JT: Persistent left superior vena cava training into the left atrium without arterial oxygen unsaturation. Am J Cardiol 1965; 16 : 273-9.

12. Cochrane AD, Marath A, Mee RB: Can a dilated coronary sinus produce left ventricular inflow obstruction? An unrecognized entity. Ann Thorac Surg 1994; 58: 1114-6.

13. Benatar A, Demanet H, Deuvaert FE: Left-ventricular inflow obstruction due to a dilated coronary sinus mimicking cot triatriatum. Thorac Cardiovasc Surg 1999; 47: 127-8.

14. Hornberger L: Anomalies of systemic and pulmonary venous drainage. In: Allan LD, Hornberger L, Sharland GK (eds): Fetal Cardiology. London: Greenwich Med Publish 2000; 103.

15. Sharland G, Cook A: Heterotaxy syndromes/ isomerism of the atrial appendages. In: Allan LD, Hornberger L, Sharland GK (eds). Fetal Cardiology. London: Greenwich Med Publish 2000; 340. 